\title{
Imaging of cerebral aneurysms: a clinical perspective
}

Nam K. Yoon ${ }^{1}$, Scott McNally ${ }^{2}$, Philipp Taussky ${ }^{1}$ and Min S. Park ${ }^{1 *}$

\begin{abstract}
Neuroimaging is a critical element in evaluating and treating patients with cerebral aneurysms. Each neuroimaging technique has unique strengths, weaknesses, and current developments. In this review, we discuss the utility of two primary noninvasive radiological techniques_computed tomography angiography (CTA) and magnetic resonance angiography (MRA) - as well as of digital subtraction angiography (DSA) for evaluation of cerebral aneurysms. These techniques allow comprehensive evaluation of aneurysm size, location, rupture status, and other imaging characteristics that guide clinicians to make appropriate and timely treatment recommendations.
\end{abstract}

Keywords: Cerebral aneurysm, Neuroimaging, Computed tomography, Magnetic resonance imaging, Digital subtraction angiography

\section{Background}

Subarachnoid hemorrhage from rupture of an intracranial aneurysm is a devastating condition associated with approximately $50 \%$ overall mortality and high survivor morbidity despite advances in treatment [1]. Although population studies have estimated the overall prevalence of intracranial aneurysms to be $3.2 \%$ [2], the overall incidence of subarachnoid hemorrhage secondary to aneurysmal rupture in a population study spanning 21 countries was relatively low at approximately 9 per 100,000 persons per year, although rates differed by country (e.g., Japan and Finland had the highest rates at 22.7 and 19.7, respectively) [3].

The International Study of Unruptured Incidental Aneurysms (ISUIA) demonstrated that the location and size of incidentally found, unruptured cerebral aneurysms were predictive of risk of future rupture [4]. Aneurysms that have already ruptured have a much higher risk of re-hemorrhage [5, 6]. Additionally, the angiomorphology of the aneurysm has also been posited to influence future hemorrhage risk factors, such as irregular domes $[7,8]$, daughter sacs [9], and low wall shear stress [10]. Thus, it is critically important to accurately characterize aneurysmal morphology to guide treatment, making

\footnotetext{
* Correspondence: neuropub@hsc.utah.edu

${ }^{1}$ Department of Neurosurgery, Clinical Neurosciences Center, University of

Utah, Salt Lake City, Utah, USA

Full list of author information is available at the end of the article
}

neuroimaging a critical element in evaluating and treating patients with cerebral aneurysms. Each neuroimaging technique has unique strengths, weaknesses, and current developments. Three major imaging methods are used for neuroimaging of cerebral aneurysms: computed tomography angiography, magnetic resonance angiography, and digital subtraction angiography. In this article, the authors review and compare imaging modalities used to evaluate intracranial aneurysms with particular focus on how each affects surgical or endovascular management.

\section{Computed tomography and CT angiography Evaluation of subarachnoid hemorrhage}

A non-contrast-enhanced CT scan is commonly used as the first-line imaging modality for evaluating subarachnoid hemorrhage (Fig. 1a). This technology is rapid, inexpensive, and widely available. In the acute setting, the higher attenuation of hemorrhage compared with surrounding parenchyma allows $100 \%$ sensitivity of subarachnoid hemorrhage if performed within the first six hours [11]. Additionally, the distribution of the hemorrhage can often predict the location of the underlying aneurysm, and CTs can determine the presence of intraventricular hemorrhage and degree of hydrocephalus, which can have significant impact on prognosis and treatment (Fig. 1a). 


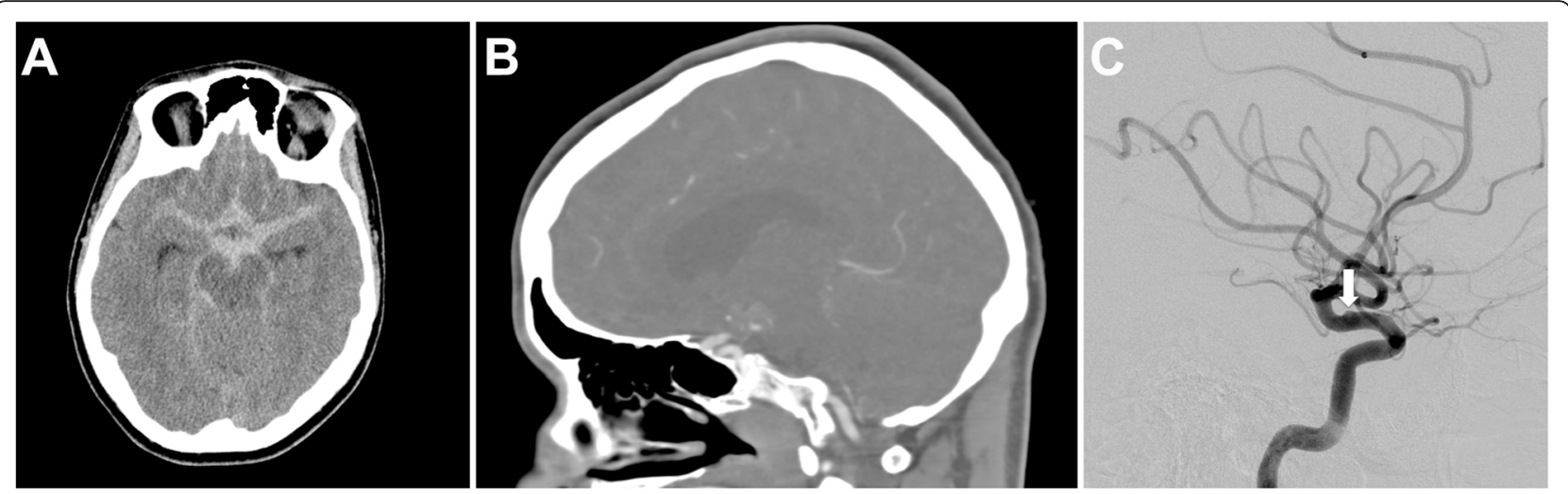

Fig. 1 Ruptured left internal carotid artery dorsal variant aneurysm. a Head CT scan demonstrating diffuse, hyperdense subarachnoid hemorrhage. b Sagittal reconstruction of a CTA of the left internal carotid artery, which did not demonstrate the aneurysm. The aneurysm was also not identified on axial or coronal projections. c Left internal carotid artery digital subtraction angiogram demonstrating a $2.6 \times 1.2-\mathrm{mm}$ blister aneurysm (white arrow)

Although CT is an excellent modality for evaluating acute subarachnoid hemorrhage, in the subacute setting, from six hours to one week after hemorrhage, the ability to detect subarachnoid hemorrhage with CT drops dramatically because of the brain's normal degradation of hemorrhagic blood products. An older study investigated the ability to detect subarachnoid hemorrhage of varying chronicity on CT and found $100 \%$ sensitivity within two days, but sensitivity dropped to $85 \%$ after five days, $50 \%$ after one week, and $30 \%$ after two weeks $[12,13]$. A more recent study with newer technology also demonstrated difficulty detecting subacute and chronic subarachnoid hemorrhage, with only $36.4 \%$ sensitivity after five days [14]. Thus, if a patient presents several days after ictus, a negative non-contrast $\mathrm{CT}$ is commonly supplemented with a lumbar puncture to evaluate for xanthochromia. Alternatively, CT angiography (CTA) has also been used to increase the detection rate of ruptured cerebral aneurysms in instances where there is a high index of clinical suspicion and a negative noncontrast CT. McCormack et al. [15] reported that a negative non-contrast $\mathrm{CT}$ followed by a negative $\mathrm{CT}$ angiogram carries a post-test probability of $99.43 \%$ of being negative for aneurysmal subarachnoid hemorrhage. The investigators argued that a lumbar puncture in this setting would carry a less than $1 \%$ probability of detecting subarachnoid hemorrhage and that patients should be informed of the low probability when lumbar puncture is offered.

\section{Detection of cerebral aneurysm}

CTA is performed on multi-detector helical CT scanners that allow multi-planar reformats, sub-millimeter slice thickness, and 3D reconstructions (Fig. 2a, b). Several investigators have demonstrated that current multidetector scanners have a spatial resolution that can reliably diagnose aneurysms greater than $4 \mathrm{~mm}$ with nearly $100 \%$ sensitivity [16-18]. A meta-analysis that surveyed studies performed on 64-slice CT scanners with sub-millimeter resolution used for detection of cerebral aneurysms showed a pooled $97 \%$ sensitivity rate for all aneurysm sizes [19]. For aneurysms $3 \mathrm{~mm}$ and smaller, however, early CT technology has been shown to be inadequate, with sensitivity numbers as low as $84 \%$ from four-channel multi-row detector CT scanners (Fig. 1b) [20]. Studies with recent technology have shown more promising results, with up to $99.6 \%$ sensitivity on 16-slice CT scanners [21]. Xing et al. [17] demonstrated an even higher sensitivity for small aneurysms on a 64-slice CT scanner than conventional nonrotational digital subtraction angiography; however, Wang et al. [22] demonstrated an $81.8 \%$ sensitivity for aneurysms smaller than $3 \mathrm{~mm}$ when performed on a 320-slice CT.

\section{Current developments}

As CT technology continues to advance with increasingly higher numbers of detectors, scans can be performed more quickly with thinner collimation, higher resolution, and better contrast bolus timing. Further increases in spatial resolution will likely allow greater sensitivity in detecting smaller aneurysms and small associated branch vessels. Time-resolved CTA, or 4DCTA, is a technique that can evaluate the flow dynamics of aneurysms by obtaining multiple acquisitions of a region of interest for a period of time. It has the ability to separate different blood flow phases and can identify aneurysms previously difficult to distinguish from surrounding vessels, such as in the case of intranidal aneurysms associated with arteriovenous malformations [23]. Additionally, specific features now discernible on electrocardiogram-gated 4D-CTA can detect aneurysm 


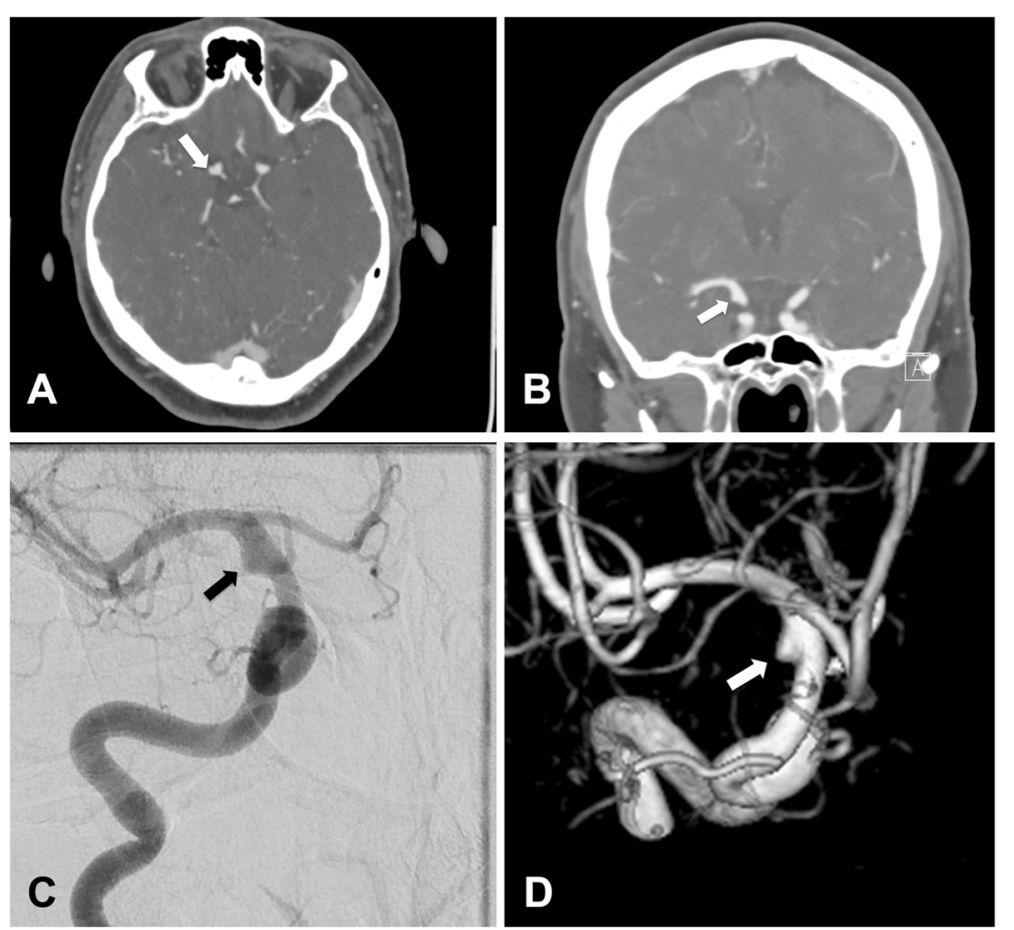

Fig. 2 Unruptured right internal carotid artery aneurysm. a Axial CT angiogram demonstrating 3-mm blister aneurysm (arrow). b Coronal CT angiogram reconstruction of the aneurysm (arrow). c Right internal carotid artery digital subtraction angiogram of the blister aneurysm (arrow). d $3 \mathrm{D}$ reconstruction during diagnostic cerebral angiogram of the aneurysm (arrow)

pulsations with the cardiac cycle and are currently being investigated for higher risk of growth and rupture [24]. Another recent development involves the use of computational fluid dynamics, which seeks to simulate blood flow within the aneurysm to calculate variables that would presumably correlate with rupture risk such as aneurysmal wall shear stress and peak wall tension. These simulations are subject to a wide range of mathematical assumptions and there has yet to be a consensus methodology for predicting rupture [25]. Certainly, computational fluid dynamics offers significant promise in the future to assist with the management of cerebral aneurysms.

\section{Magnetic resonance imaging and MR angiography \\ Evaluation of subarachnoid hemorrhage}

The use of MRI for evaluation of cerebral aneurysms is continually and rapidly evolving. MRI has advantages over CTA in that it does not use ionizing radiation and offers the ability to obtain images without the need for administration of intravenous contrast agents. Fluidattenuated inversion recovery (FLAIR) sequences produce strong T2 weighting while suppressing the cerebrospinal fluid (CSF) signal and can be particularly useful for detecting subarachnoid hemorrhage, especially in the subacute period. In a study of patients with acute and subacute subarachnoid hemorrhage, da Rocha et al. [26] demonstrated a $100 \%$ sensitivity for detection of subarachnoid hemorrhage with FLAIR compared with a $66 \%$ sensitivity on CT. Gradient-echo T2*-weighted imaging was found to be superior to FLAIR for detection of subarachnoid hemorrhage of even older age [14]. However, because of the higher cost and longer acquisition times, MRI is not considered the initial diagnostic test of choice and is usually reserved for patients with a high pre-test probability of aneurysmal subarachnoid hemorrhage and a negative initial work-up with CT scans and/or cerebral angiography. It has the added benefit of screening for other pathologies that may present with similar symptoms, some of which may also produce hyperintense sulcal signal on FLAIR such as meningitis, meningeal carcinomatosis, leptomeningeal metastasis, subdural hematoma, adjacent neoplasms, and dural venous thrombosis.

\section{MRI/MRA aneurysm detection}

The use of time-of-flight (TOF) sequences on MRA eliminates the need for administration of contrast agents, which may be contraindicated in patients with renal failure or contrast allergy (Fig. 3a, b and Fig. 4). A meta-analysis of MRA studies for evaluating aneurysms demonstrated that contrast-enhanced MRA (CE-MRA) and TOF had similar sensitivity for the 

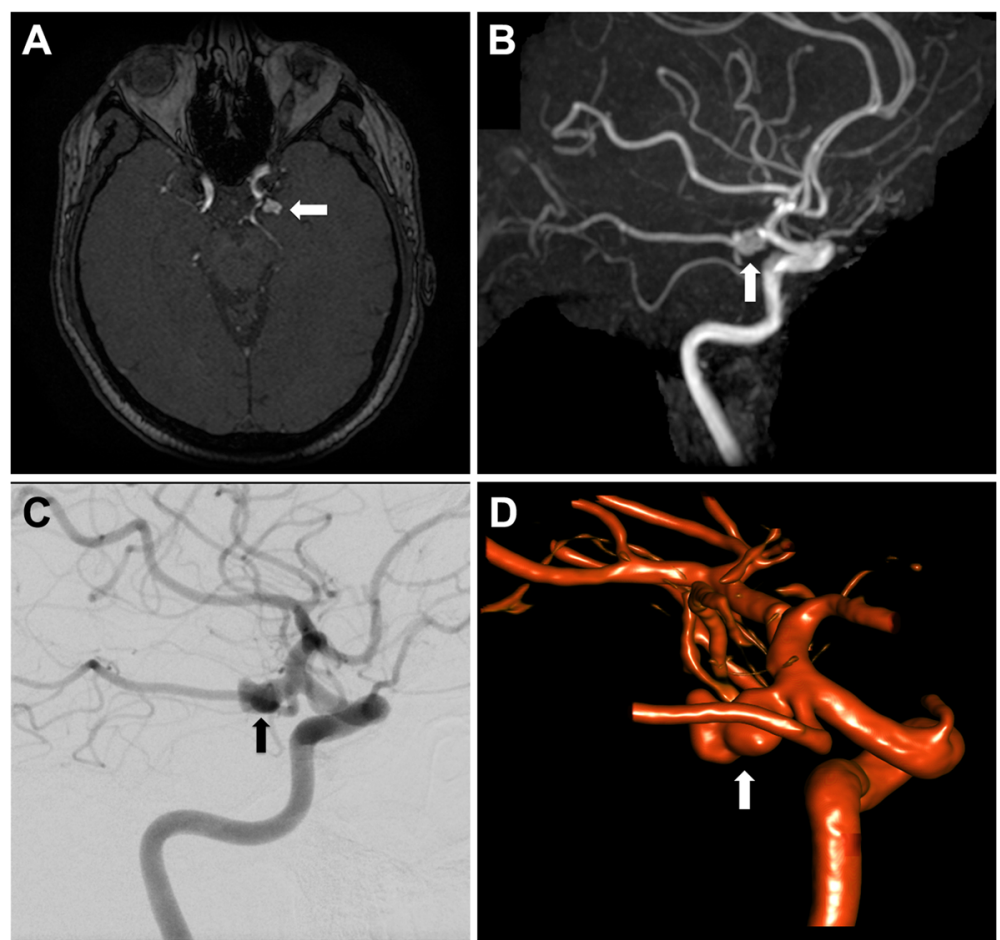

Fig. 3 Unruptured $8 \times 4$-mm left posterior communicating artery aneurysm. a 3D TOF MRA demonstrating irregular 8-mm aneurysm (arrow). b 3D MRA reconstruction of the aneurysm (arrow). c Left internal carotid artery digital subtraction angiogram of the aneurysm (arrow). d 3D reconstruction during diagnostic cerebral angiogram of the posterior communicating artery aneurysm (arrow)

detection of aneurysms [27]. CE-MRA, however, was found to be superior to TOF sequences because of its ability to eliminate flow-related artifacts and spin saturation [28]. Although CE-MRA had a lower spatial resolution compared with $\mathrm{CT}$ angiography $(0.7 \times 0.7 \times$ 0.8 versus $0.35 \times 0.35 \times 0.8 \mathrm{~mm}$ ), the images were qualitatively graded to be comparable with similar diagnostic results (Fig. 4) [29]. Small branch vessels were also reportedly better visualized on $3 \mathrm{~T}$ MRA

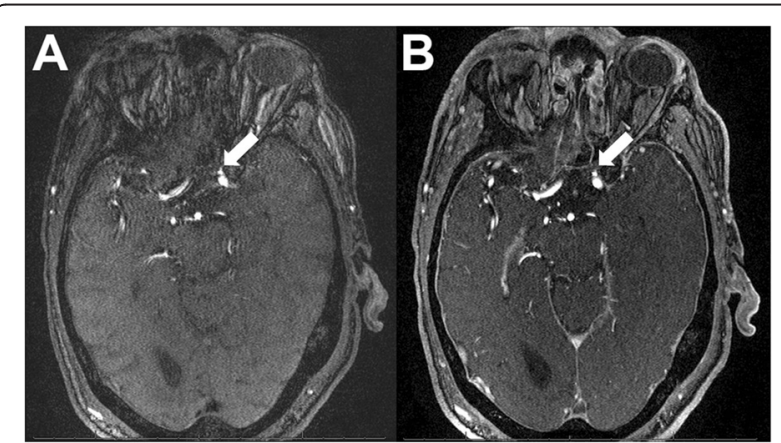

Fig. 4 MRA scan of aneurysms. a Axial TOF MRA demonstrates a left ophthalmic artery aneurysm (arrow). Although it avoids the use of contrast, the longer acquisition time introduces some motion artifact. b Contrast-enhanced axial MRA in the same patient with less motion artifact of the aneurysm (arrow) over CTA, because of the lack of venous contrast contamination and in spite of lower spatial resolution [30]. Despite these findings, MRA is generally not the diagnostic test of choice for the evaluation of cerebral aneurysms in the acute setting and is reserved as a screening test or for follow-up, where avoiding repeated ionizing radiation and/or contrast nephropathy is important.

As MRA technology continues to improve, spatial resolution and sensitivity to smaller aneurysms is also expected to improve. With current sequences, the spatial resolution at $1.5 \mathrm{~T}$ is often as low as $1 \mathrm{~mm}$ [31], whereas at $3 \mathrm{~T}$ the spatial resolution can be as low as $0.6-$ $0.7 \mathrm{~mm}$ while preserving the signal-to-noise ratio [32]. Radiologists also unanimously report significantly better visualization of aneurysms at $3 \mathrm{~T}$. A meta-analysis by Sailer et al. [27] that evaluated the sensitivity of MRA for detection of cerebral aneurysms found a trend towards significance in better detection of smaller aneurysms on $3 \mathrm{~T}$ versus $1.5 \mathrm{~T}$ scanners $(p=0.054)$. This study reported a pooled sensitivity of $1.5 \mathrm{~T}$ and $3 \mathrm{~T}$ scanners of approximately $95 \%$ for all aneurysms. Although the above study showed that $90 \%$ of aneurysms that were missed were smaller than $5 \mathrm{~mm}$ in size, the pooled sensitivity for detecting aneurysms smaller than $3 \mathrm{~mm}$ was still $86 \%$. 


\section{Future developments}

Although ISUIA argued that aneurysms smaller than $7 \mathrm{~mm}$ have very low rates of hemorrhage [4], the majority of ruptured aneurysms tend to be small [33]. There is a current unmet need to identify signs of high rupture risk in these small aneurysms. The pathophysiology of aneurysm growth, wall thinning, and rupture involves multiple histologic changes including endothelial disruption, collagen loss, and inflammatory cell migration [34]. Current imaging cannot detect these changes, but contrast agents could permeate through the disrupted endothelium into the aneurysm wall or even the surrounding CSF. Researchers have recently developed black-blood T1-weighted MRI sequences that help identify rupture site in patients with aneurysmal subarachnoid hemorrhage by demonstrating the inflamed, enhancing wall of the recently ruptured aneurysm. These techniques can identify the causative rupture site in patients with multiple intracranial aneurysms, allowing for more accurate and timely treatment [35].

Aneurysmal wall enhancement correlates with previous rupture, with some enhancement (strong or weak) identified in $98.4 \%$ of ruptured aneurysms and no enhancement identified in $81.9 \%$ of unruptured aneurysms [36]. Aneurysm wall permeability can also be quantified using dynamic contrast-enhanced (DCE)-MRI and the perfusion parameter $\mathrm{K}^{\text {trans }}$, which is a size-independent predictor of rupture risk [37]. Additional imaging protocols are being developed to help further characterize aneurysms and identify characteristics associated with rupture risk. Highfield-strength $7 \mathrm{~T}$ MRI scanners have an extremely high signal-to-noise ratio, allowing the spatial resolution needed to detect variations in aneurysm wall thickness, which may be a marker of rupture risk [38]. Other protocols can define aneurysmal wall motion abnormalities associated with higher risk of rupture [39]. Another advanced imaging technique combines MRA with postprocessing algorithms from computational fluid dynamics to create 4D flow modeling [40, 41]. Time-resolved MRA, or 4D MRA, directly measures flow velocities and shear stresses within an aneurysm [42]. This may allow for the identification of the inflow zone of aneurysms and may guide endovascular treatment $[43,44]$.

\section{Digital subtraction angiography}

Digital subtraction angiography (DSA) is typically performed using femoral catheterization with a biplane unit with rotational/3-dimensional capabilities. The catheter is guided to each of the major cerebral arteries, and iodinated contrast is injected while obtaining various $2 \mathrm{D}$ views, with rotational angiography used to create a 3dimensional model of the vessels.

Considered the reference standard for evaluating intracranial aneurysms, DSA has high spatial resolution to identify aneurysms that may be missed on CTA or MRA (Figs. 1, 2 and 3). It also has the temporal resolution to separately evaluate the arterial, capillary, and venous phases of the injection. Similar to CTA, DSA requires the use of ionizing radiation and iodinated contrast. In addition, this procedure is invasive and requires operator expertise to guide the catheter to the major intracranial vessels while minimizing the risk of stroke, vascular injury, femoral artery occlusion, or retroperitoneal hematoma. A review of eight prospective and seven retrospective studies to determine the complication rate of DSA for evaluation of carotid stenosis demonstrated a mortality rate of $0.06 \%$ and a rate of permanent neurologic deficit from stroke of $1 \%$ [45]. Another study showed greater re-hemorrhage rates when DSA was used to evaluate ruptured aneurysms within the first six hours post ictus [46]; however, as these are operatordependent procedures, overall risks may be related to operator expertise, with reports of complication rates of DSA for various indications below $0.3 \%$ [47].

In addition to conventional DSA, 3-dimensional rotational angiography (3DRA) further increases the sensitivity of small aneurysms less than $3 \mathrm{~mm}$ in size (Figs. 1 and 2). van Rooij et al. [48] demonstrated that the addition of 3DRA identified 94 additional aneurysms in the evaluation of 350 target aneurysms initially screened by CTA/MRA. The mean size of these additional aneurysms was $3.54 \mathrm{~mm}$, with the smallest detected aneurysm at $0.5 \mathrm{~mm}$. Twentyseven of these 94 aneurysms (mean size $1.94 \mathrm{~mm}$ ) were even missed on DSA without 3DRA. Compared with 64and 256-detector-row CTA, 3DRA is superior in detecting aneurysms $3 \mathrm{~mm}$ and smaller [49] and should be used when CT and/or MRI fails to demonstrate an aneurysm in the setting of spontaneous subarachnoid hemorrhage. Even when DSA fails to demonstrate an aneurysm in the setting of spontaneous subarachnoid hemorrhage, delayed repeat imaging is indicated. One literature review identified 37 aneurysms out of 368 angiography-negative subarachnoid hemorrhage patients on delayed, repeat DSA from one to six weeks after their initial study [50]. The higher resolution of DSA also allows for better neck characterization to guide endovascular versus open surgical treatment and better visualization of associated branch vessels [30].

\section{Conclusion}

It is important to accurately determine the size, morphology, location, and rupture status of a cerebral aneurysm and/or to identify specific imaging characteristics that may portend a higher risk of rupture so that potential treatment can be guided more accurately. While CT, MRI, and DSA are excellent tools at our disposal to identify subarachnoid hemorrhage and cerebral aneurysms, future advances will likely allow for faster, safer, and more accurate identification of cerebral aneurysms. 


\section{Competing interests}

The authors declare that they have no competing interests.

\section{Authors' contributions}

All authors read and approved the final manuscript.

\section{Author details}

'Department of Neurosurgery, Clinical Neurosciences Center, University o Utah, Salt Lake City, Utah, USA. ${ }^{2}$ Department of Radiology, University of Utah Health Care, Salt Lake City, Utah, USA.

\section{Received: 30 November 2015 Accepted: 2 February 2016 Published online: 15 March 2016}

\section{References}

1. Hop JW, Rinkel GJ, Algra A, van Gijn J. Case-fatality rates and functional outcome after subarachnoid hemorrhage: a systematic review. Stroke. 1997:28:660-4

2. Vlak MH, Algra A, Brandenburg R, Rinkel GJ. Prevalence of unruptured intracranial aneurysms, with emphasis on sex, age, comorbidity, country, and time period: a systematic review and meta-analysis. Lancet Neurol. 2011;10:626-36

3. de Rooij NK, Linn FH, van der Plas JA, Algra A, Rinkel GJ. Incidence of subarachnoid haemorrhage: a systematic review with emphasis on region, age, gender and time trends. J Neurol Neurosurg Psychiatry. 2007;78:1365-72

4. Wiebers DO, Whisnant JP, Huston 3rd J, Meissner I, Brown Jr RD, Piepgras DG, et al. Unruptured intracranial aneurysms: natural history, clinical outcome, and risks of surgical and endovascular treatment. Lancet. 2003;362:103-10

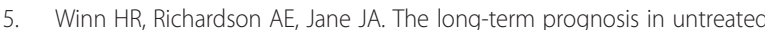
cerebral aneurysms: I. The incidence of late hemorrhage in cerebral aneurysm: a 10-year evaluation of 364 patients. Ann Neurol. 1977;1:358-70.

6. Eskesen $V$, Rosenorn J, Schmidt $K$. The impact of rebleeding on the life time probabilities of different outcomes in patients with ruptured intracranial aneurysms. A theoretical evaluation. Acta Neurochir (Wien). 1988;95:99-101.

7. Raghavan ML, Ma B, Harbaugh RE. Quantified aneurysm shape and rupture risk. J Neurosurg. 2005;102:355-62.

8. Dhar S, Tremmel M, Mocco J, Kim M, Yamamoto J, Siddiqui AH, et al. Morphology parameters for intracranial aneurysm rupture risk assessment. Neurosurgery. 2008;63:185-96. discussion 196-197.

9. UCAS Japan Investigators. The natural course of unruptured cerebral aneurysms in a Japanese cohort. N Engl J Med. 2012:366:2474-82.

10. Boussel L, Rayz V, McCulloch C, Martin A, Acevedo-Bolton G, Lawton M, et al. Aneurysm growth occurs at region of low wall shear stress: patientspecific correlation of hemodynamics and growth in a longitudinal study. Stroke. 2008;39:2997-3002

11. Boesiger BM, Shiber JR. Subarachnoid hemorrhage diagnosis by computed tomography and lumbar puncture: are fifth generation CT scanners better at identifying subarachnoid hemorrhage? J Emerg Med. 2005;29:23-7.

12. Perry JJ, Stiell IG, Sivilotti ML, Bullard MJ, Emond M, Symington C, et al. Sensitivity of computed tomography performed within six hours of onset of headache for diagnosis of subarachnoid haemorrhage: prospective cohort study. BMJ. 2011;343:d4277.

13. van Gijn J, van Dongen KJ. The time course of aneurysmal haemorrhage on computed tomograms. Neuroradiology. 1982;23:153-6.

14. Yuan MK, Lai PH, Chen JY, Hsu SS, Liang HL, Yeh LR, et al. Detection of subarachnoid hemorrhage at acute and subacute/chronic stages: comparison of four magnetic resonance imaging pulse sequences and computed tomography. J Chin Med Assoc. 2005;68:131-7.

15. McCormack RF, Hutson A. Can computed tomography angiography of the brain replace lumbar puncture in the evaluation of acute-onset headache after a negative noncontrast cranial computed tomography scan? Acad Emerg Med. 2010;17:444-51.

16. Uysal E, Yanbuloglu B, Erturk M, Kilinc BM, Basak M. Spiral CT angiography in diagnosis of cerebral aneurysms of cases with acute subarachnoid hemorrhage. Diagn Interv Radiol. 2005;11:77-82.

17. Xing W, Chen W, Sheng J, Peng Y, Lu J, Wu X, et al. Sixty-four-row multislice computed tomographic angiography in the diagnosis and characterization of intracranial aneurysms: comparison with 3D rotational angiography. World Neurosurg. 2011;76:105-13.
18. McKinney AM, Palmer CS, Truwit CL, Karagulle A, Teksam M. Detection of aneurysms by 64-section multidetector $C T$ angiography in patients acutely suspected of having an intracranial aneurysm and comparison with digital subtraction and 3D rotational angiography. AJNR Am J Neuroradiol. 2008:29:594-602.

19. Guo W, He XY, Li XF, Qian DX, Yan JQ, Bu DL, et al. Meta-analysis of diagnostic significance of sixty-four-row multi-section computed tomography angiography and three-dimensional digital subtraction angiography in patients with cerebral artery aneurysm. J Neurol Sci. 2014;346:197-203.

20. Teksam M, McKinney A, Casey S, Asis M, Kieffer S, Truwit CL. Multi-section CT angiography for detection of cerebral aneurysms. AJNR Am J Neuroradiol. 2004;25:1485-92.

21. Prestigiacomo CJ, Sabit A, He W, Jethwa P, Gandhi C, Russin J. Three dimensional $C T$ angiography versus digital subtraction angiography in the detection of intracranial aneurysms in subarachnoid hemorrhage. J Neurointerv Surg. 2010;2:385-9.

22. Wang H, Li W, He H, Luo L, Chen C, Guo Y. 320-detector row CT angiography for detection and evaluation of intracranial aneurysms: comparison with conventional digital subtraction angiography. Clin Radiol. 2013;68:e15-20

23. Chandran A, Radon M, Biswas S, Das K, Puthuran M, Nahser H: Novel use of 4DCTA in imaging of intranidal aneurysms in an acutely ruptured arteriovenous malformation: is this the way forward? J Neurointerv Surg. (in press).

24. Hayakawa M, Tanaka T, Sadato A, Adachi K, Ito K, Hattori N, et al. Detection of pulsation in unruptured cerebral aneurysms by ECG-gated 3D-CT angiography (4D-CTA) with 320-row area detector CT (ADCT) and follow-up evaluation results: assessment based on heart rate at the time of scanning. Clin Neuroradiol. 2014:24:145-50.

25. Berg P, Roloff C, Beuing O, Voss S, Sugiyama S, Aristokleous N, et al. The Computational Fluid Dynamics Rupture Challenge 2013-Phase II: Variability of hemodynamic simulations in two intracranial aneurysms. J Biomech Eng. 2015;137:121008.

26. da Rocha AJ, da Silva CJ, Gama HP, Baccin CE, Braga FT, de Araújo Cesare F, et al. Comparison of magnetic resonance imaging sequences with computed tomography to detect low-grade subarachnoid hemorrhage: Role of fluid-attenuated inversion recovery sequence. J Comput Assist Tomogr. 2006;30:295-303.

27. Sailer AM, Wagemans BA, Nelemans PJ, de Graaf R, van Zwam WH. Diagnosing intracranial aneurysms with MR angiography: systematic review and meta-analysis. Stroke. 2014;45:119-26.

28. Nael K, Villablanca JP, Saleh R, Pope W, Nael A, Laub G, et al. Contrastenhanced MR angiography at $3 \mathrm{~T}$ in the evaluation of intracranial aneurysms: a comparison with time-of-flight MR angiography. AJNR Am J Neuroradiol. 2006;27:2118-21.

29. Nael K, Villablanca JP, Mossaz L, Pope W, Juncosa A, Laub G, et al. 3-T contrast-enhanced MR angiography in evaluation of suspected intracranial aneurysm: comparison with MDCT angiography. AJR Am J Roentgenol. 2008;190:389-95.

30. Goto M, Kunimatsu A, Shojima M, Mori H, Abe O, Aoki S, et al. Depiction of branch vessels arising from intracranial aneurysm sacs: Time-of-flight MR angiography versus CT angiography. Clin Neurol Neurosurg. 2014;126:177-84.

31. Schellinger PD, Richter G, Kohrmann M, Dorfler A. Noninvasive angiography (magnetic resonance and computed tomography) in the diagnosis of ischemic cerebrovascular disease. Techniques and clinical applications. Cerebrovasc Dis. 2007;24 Suppl 1:16-23.

32. Bernstein MA, Huston 3rd J, Lin C, Gibbs GF, Felmlee JP. High-resolution intracranial and cervical MRA at 3.0 T: technical considerations and initial experience. Magn Reson Med. 2001;46:955-62

33. Forget Jr TR, Benitez R, Veznedaroglu E, Sharan A, Mitchell W, Silva M, et al. A review of size and location of ruptured intracranial aneurysms. Neurosurgery. 2001;49:1322-5. discussion 1325-1326.

34. Frosen J, Piippo A, Paetau A, Kangasniemi M, Niemela M, Hernesniemi J, et al. Remodeling of saccular cerebral artery aneurysm wall is associated with rupture: histological analysis of 24 unruptured and 42 ruptured cases. Stroke. 2004;35:2287-93.

35. Matouk CC, Mandell DM, Gunel M, Bulsara KR, Malhotra A, Hebert R, et al. Vessel wall magnetic resonance imaging identifies the site of rupture in patients with multiple intracranial aneurysms: proof of principle. Neurosurgery. 2013;72:492-6. discussion 496

36. Nagahata S, Nagahata M, Obara M, Kondo R, Minagawa N, Sato S, et al. Wall enhancement of the intracranial aneurysms revealed by magnetic 
resonance vessel wall imaging using three-dimensional turbo spin-echo sequence with motion-sensitized driven-equilibrium: A sign of ruptured aneurysm? Clin Neuroradiol. (in press).

37. Vakil P, Ansari SA, Cantrell CG, Eddleman CS, Dehkordi FH, Vranic J, et al. Quantifying intracranial aneurysm wall permeability for risk assessment using dynamic contrast-enhanced MRI: A pilot study. AJNR Am J Neuroradiol. 2015;36:953-9.

38. Kleinloog R, Korkmaz E, Zwanenburg JJ, Kuijf HJ, Visser F, Blankena R, et al. Visualization of the aneurysm wall: a 7.0-tesla magnetic resonance imaging study. Neurosurgery. 2014;75:614-22. discussion 622.

39. Vanrossomme AE, Eker OF, Thiran JP, Courbebaisse GP, Zouaoui Boudjeltia K. Intracranial aneurysms: Wall motion analysis for prediction of rupture. AJNR Am J Neuroradiol. 2015;36:1796-1802.

40. Cebral JR, Vazquez M, Sforza DM, Houzeaux G, Tateshima S, Scrivano E, et al. Analysis of hemodynamics and wall mechanics at sites of cerebral aneurysm rupture. J Neurointerv Surg. 2015;7:530-6.

41. Schnell S, Ansari SA, Vakil P, Wasielewski M, Carr ML, Hurley MC, et al. Threedimensional hemodynamics in intracranial aneurysms: influence of size and morphology. J Magn Reson Imaging. 2014;39:120-31.

42. Boussel L, Rayz V, Martin A, Acevedo-Bolton G, Lawton MT, Higashida R, et al. Phase-contrast magnetic resonance imaging measurements in intracranial aneurysms in vivo of flow patterns, velocity fields, and wall shear stress: comparison with computational fluid dynamics. Magn Reson Med. 2009;61:409-17.

43. Schneiders JJ, Marquering HA, van Ooij P, van den Berg R, Nederveen AJ, Verbaan $\mathrm{D}$, et al. Additional value of intra-aneurysmal hemodynamics in discriminating ruptured versus unruptured intracranial aneurysms. AJNR Am J Neuroradiol. 2015;36:1920-6.

44. Futami K, Sano H, Misaki K, Nakada M, Ueda F, Hamada J. Identification of the inflow zone of unruptured cerebral aneurysms: comparison of $4 D$ flow MRI and 3D TOF MRA data. AJNR Am J Neuroradiol. 2014;35:1363-70.

45. Hankey GJ, Warlow CP, Sellar RJ. Cerebral angiographic risk in mild cerebrovascular disease. Stroke. 1990;21:209-22.

46. Inagawa T, Kamiya K, Ogasawara H, Yano T. Rebleeding of ruptured intracranial aneurysms in the acute stage. Surg Neurol. 1987;28:93-9.

47. Fifi JT, Meyers PM, Lavine SD, Cox V, Silverberg L, Mangla S, et al. Complications of modern diagnostic cerebral angiography in an academic medical center. J Vasc Interv Radiol. 2009;20:442-7.

48. van Rooij WJ, Sprengers ME, de Gast AN, Peluso JP, Sluzewski M. 3D rotational angiography: the new gold standard in the detection of additional intracranial aneurysms. AJNR Am J Neuroradiol. 2008;29:976-9.

49. Bechan RS, van Rooij SB, Sprengers ME, Peluso JP, Sluzewski M, Majoie CB, et al. $C T$ angiography versus $3 D$ rotational angiography in patients with subarachnoid hemorrhage. Neuroradiology. 2015;57:1239-46.

50. Bakker NA, Groen RJ, Foumani M, Uyttenboogaart M, Eshghi OS, Metzemaekers JD, et al. Repeat digital subtraction angiography after a negative baseline assessment in nonperimesencephalic subarachnoid hemorrhage: a pooled data meta-analysis. J Neurosurg. 2014;120:99-103.

\section{Submit your next manuscript to BioMed Central and we will help you at every step:}

- We accept pre-submission inquiries

- Our selector tool helps you to find the most relevant journal

- We provide round the clock customer support

- Convenient online submission

- Thorough peer review

- Inclusion in PubMed and all major indexing services

- Maximum visibility for your research

Submit your manuscript at www.biomedcentral.com/submit

) Biomed Central 